\title{
The attentional blink with targets in different spatial locations
}

\author{
TROY A. W. VISSER and SAMANTHA M. ZUVIC \\ University of British Columbia, Vancouver, British Columbia, Canada \\ WALTER F. BISCHOF \\ University of Alberta, Edmonton, Alberta, Canada \\ and \\ VINCENT DI LOLLO \\ University of British Columbia, Vancouver, British Columbia, Canada
}

\begin{abstract}
When two targets (T1 and T2) are displayed in rapid succession, accuracy of T2 identification varies as a function of the temporal lag between the targets (attentional blink, AB). In some studies, performance has been found to be most impaired at Lag 1-namely, when T2 followed T1 directly. In other studies, T2 performance at Lag 1 has been virtually unimpaired (Lag 1 sparing). In the present work, we examined how Lag 1 sparing is affected by attentional switches between targets displayed in the same location or in different locations. We found that Lag 1 sparing does not occur when a spatial shift is required between $\mathrm{T} 1$ and $\mathrm{T} 2$. This suggests that attention cannot be switched to a new location while the system is busy processing another stimulus. The results are explained by a modified version of an attentional gating model (Chun \& Potter, 1995; Shapiro \& Raymond, 1994).
\end{abstract}

There is a cost to attending to two visual targets presented in rapid temporal sequence. Identification is almost flawless for the first target (T1) but is impaired for the second (T2). This second-target deficit, known as the attentional blink (AB), has been studied with a paradigm called rapid serial visual presentation (RSVP), in which the two targets are inserted in a stream of distractors (e.g., Chun \& Potter, 1995; Raymond, Shapiro, \& Arnell, 1992). All items in the RSVP stream are presented at the same spatial location at a rate of approximately 10 items $/ \mathrm{sec}$. The temporal lag between the two targets is manipulated by varying the number of intervening distractors. The $\mathrm{AB}$ deficit has also been studied with another paradigm, called the two-target paradigm, in which there are no distractors, and the two targets, each followed by a mask, are usually displayed at different screen locations (e.g., Duncan, Ward, \& Shapiro, 1994; Ward, Duncan, \& Shapiro, 1997). In this paradigm, the lag between the targets is manipulated by varying the duration of an intervening blank interval.

The magnitude of the $A B$ deficit is known to vary as a function of the temporal lag between the two targets. Two distinct patterns of performance across lags have been

This work was supported by grants from the Natural Sciences and Engineering Research Council of Canada to W. F. Bischof and V. Di Lollo. The authors thank James $C$. Johnston and William S. Maki for commenting on an earlier version of this article. Correspondence should be addressed to T. A. W. Visser, Department of Psychology, University of British Columbia, 2136 West Mall, Vancouver, BC, V6T 1Z4 Canada (e-mail: tvisser@interchange.ubc.ca). found. In some experiments, the function was monotonic, with the largest deficit occurring when T2 was presented directly after T1 (an interval known as Lag 1 ) and performance improving steadily at longer lags (Duncan, Martens, \& Ward, 1997; Joseph, Chun, \& Nakayama, 1997). In other experiments, the function was U-shaped, with the lowest level of $\mathrm{T} 2$ identification occurring at Lag 2 or 3 (i.e., 200-300 msec after T1). In these experiments, performance was relatively high at Lag 1, dropped substantially at Lags 2 and 3, and then recovered at longer lags. Except for the substantial difference in Lag 1 performance, monotonic and U-shaped functions tend to be quite similar (e.g., Peterson \& Juola, 1997). Following the practice of Potter, Chun, Banks, and Muckenhoupt (1998), we refer to the instances of higher performance at Lag 1 as Lag 1 sparing.

Lag 1 sparing is commonly ascribed to the sluggish closing of an attentional gate (Chun \& Potter, 1995; Shapiro \& Raymond, 1994). The gate is said to open rapidly on presentation of TI but to close slowly, thus permitting the next item in the stream (i.e., the item in the Lag 1 position) to enter a higher processing stage along with $\mathrm{T} 1$. Thus, if $\mathrm{T} 2$ arrives while the attentional gate is still open, both targets become part of the same attentional episode, both gain access to high-level processing mechanisms, and Lag 1 sparing ensues. If $\mathrm{T} 2$ arrives after the gate has closed, it is delayed at a lower processing level, where it is subject to passive decay and to backward masking by subsequent items, and Lag 1 sparing does not occur.

Although plausible, the attentional gating hypothesis has a major drawback: It cannot account for many in- 
stances in which Lag 1 sparing fails to occur. According to the gating hypothesis, Lag 1 sparing should invariably take place, provided that $\mathrm{T} 1$ and $\mathrm{T} 2$ are presented in close temporal contiguity. Yet several experiments in which the two targets were presented in rapid succession failed to reveal Lag 1 sparing (e.g., Duncan et al., 1997; Jolicoeur, 1998; Joseph et al., 1997; Moore, Egeth, Berglan, \& Luck, 1996). This indicates that temporal contiguity alone is insufficient for a complete account of Lag 1 sparing.

Why is Lag 1 sparing found in some experiments but not in others? In a recent survey of the $\mathrm{AB}$ literature (Visser, Bischof, \& Di Lollo, in press), we discovered that, with one possible exception (Duncan et al., 1994, Experiment 1), Lag 1 sparing is never found when the two targets are displayed in different spatial locations. This is true not only for the two-target paradigm, in which targets are usually presented in different locations (Duncan et al., 1997; Moore et al., 1996), but also for the RSVP paradigm. For example, using the RSVP paradigm, Breitmeyer, Ehrenstein, Pritchard, Hiscock, and Crisan (1999) found substantial Lag 1 sparing when all items were displayed in the same location but not when they were displayed haphazardly in multiple locations. A comparable outcome has been reported by Joseph et al. (1997).

On this evidence, spatial switching seems to play an important role in the failure of Lag 1 sparing. The evidence, however, is illustrative rather than comprehensive, because earlier experiments examined only a subset of the possible combinations of the locations of the two targets in relation to a central RSVP stream of distractors. Joseph et al. (1997) considered only the case in which TI was presented at the same central location as the distractor stream, and T2 was presented in an unpredictable eccentric location. Breitmeyer et al. (1999), on the other hand, displayed T1 and T2 either in the same central location as the RSVP stream or both eccentrically in different locations.

The present experiment was designed to consider the remaining possibilities. We examined the cases of an eccentric $\mathrm{T} 1$ and a central $\mathrm{T} 2$, as well as the case in which $\mathrm{T} 1$ and $\mathrm{T} 2$ are both presented at the same eccentric location. We compared these cases with the more common case in which T1 and T2 are both central, as in the conventional RSVP paradigm, and with the case in which Tl was presented centrally and was followed by an eccentric T2, as in Joseph et al. (1997). Two main findings emerged. First, the outcome revealed that temporal contiguity between the targets is necessary but not sufficient for Lag I sparing. Second, Lag 1 sparing is eliminated only when a spatial switch is required between $\mathrm{T} 1$ and $\mathrm{T} 2$. Together, these results favor an account in terms of a sluggish, location-specific attentional gate controlling the transfer of information from an earlier to a later processing stage.

\section{METHOD}

\section{Observers}

Twenty-four undergraduate students from the University of British Columbia participated for class credit. All had normal or corrected-to-normal vision based on self-report. None of the observers had participated in any other experiment concerning the $A B$.

\section{Apparatus and Stimuli}

All stimuli were displayed on a Tektronix 608 oscilloscope equipped with P15 phosphor. The viewing distance, set by a headrest, was $57 \mathrm{~cm}$. All stimuli subtended approximately $1^{\circ}$ of visual angle and had a luminance of $25 \mathrm{~cd} / \mathrm{m}^{2}$, as measured by a Minolta LS-100 luminance meter. The distractor items were digits (0-9), and the target items were letters from the English alphabet. The background and surrounding visual field were dark, except for dim illumination of the keyboard.

\section{Procedure}

At the beginning of each trial a small fixation dot was presented in the center of the screen, indicating where the RSVP items would be displayed. The observers initiated each trial by pressing the space bar. After a 500-msec delay, the RSVP stream was presented. Each item was displayed for $32 \mathrm{msec}$, and was separated from the next item by an interstimulus interval (ISI) of $68 \mathrm{msec}$, yielding a presentation rate of 10 items $/ \mathrm{sec}$. On any given trial, the distractors were selected randomly with replacement from the set of digits, with the constraint that the selected digit was not one of the two immediately preceding items. Letter targets were selected randomly without replacement from all letters of the English alphabet, except for $I, O, Q$, and $Z$ (which are visually similar to $1,0,7$, and 2 ). The number of distractors that preceded the first target varied randomly on each trial from 7 to 10 .

Most $A B$ studies have examined a range of five or more lags between $T 1$ and $T 2$. The present work was concerned primarily with Lag 1 sparing, which occurs when $\mathrm{T} 2$ is presented directly after $\mathrm{T} 1$. Therefore, in the interest of maximizing the number of withinsubjects factors relevant to Lag 1 sparing in a single experimental session, we opted to examine only two other lags beyond Lag 1: a lag of $300 \mathrm{msec}$, at which the $A B$ deficit is usually at a maximum, and a lag of $700 \mathrm{msec}$, at which the $\mathrm{AB}$ deficit has usually subsided. Thus, within a given RSVP stream, $T 2$ was presented 100,300 , or 700 msec after the onset of $\mathrm{Tl}$ ( $\operatorname{Lag} 1,3$, or 7 ) and was always followed by a single digit, which acted as a mask, at a stimulus onset asynchrony (SOA) of $100 \mathrm{msec}$. The observers were required to identify the two letters in any order by typing them into the keyboard. Then, the fixation dot reappeared to indicate that the next trial was ready to begin.

The observers participated in four blocks of trials in a single $1-h$ session. As illustrated in Figure 1, the four blocks differed with respect to the positioning of the targets relative to the stream. In Condition $\mathrm{CC}$, both targets were displayed at the same central location as the distractor stream. In Condition EE, both targets appeared at one of four eccentric locations, above, below, to the right of, or to the left of the central distractor stream. The center-to-center separation of eccentric and central locations was $1^{\circ}$. In Condition EE, both targets were displayed in the same location, selected randomly on each trial, as explained below. In Condition CE, T1 was presented at the same location as the central stream, and $\mathrm{T} 2$ was presented in one of the four eccentric positions. Finally, in Condition EC, T1 appeared in one of the four eccentric positions, and $\mathrm{T} 2$ was presented at the same location as the central stream. The location of the eccentric targets (above, below, left of, or right of center) was chosen randomly on each trial, with the restriction that each of the four locations was chosen an equal number of times within a session. In Conditions EE, CE, and EC, the eccentric target was always followed by an item, which acted as a mask, presented in the same location at an SOA of $100 \mathrm{msec}$. The trailing item could be either T2 or a digit, depending on the condition, as illustrated in Figure 1.

Each block consisted of 96 trials in which the second target was presented equally often at each of the three lags. Before the first block of trials, the observers completed 15 practice trials and then 5 additional practice trials at the beginning of each subsequent block. The observers were allowed a brief rest period between blocks. 


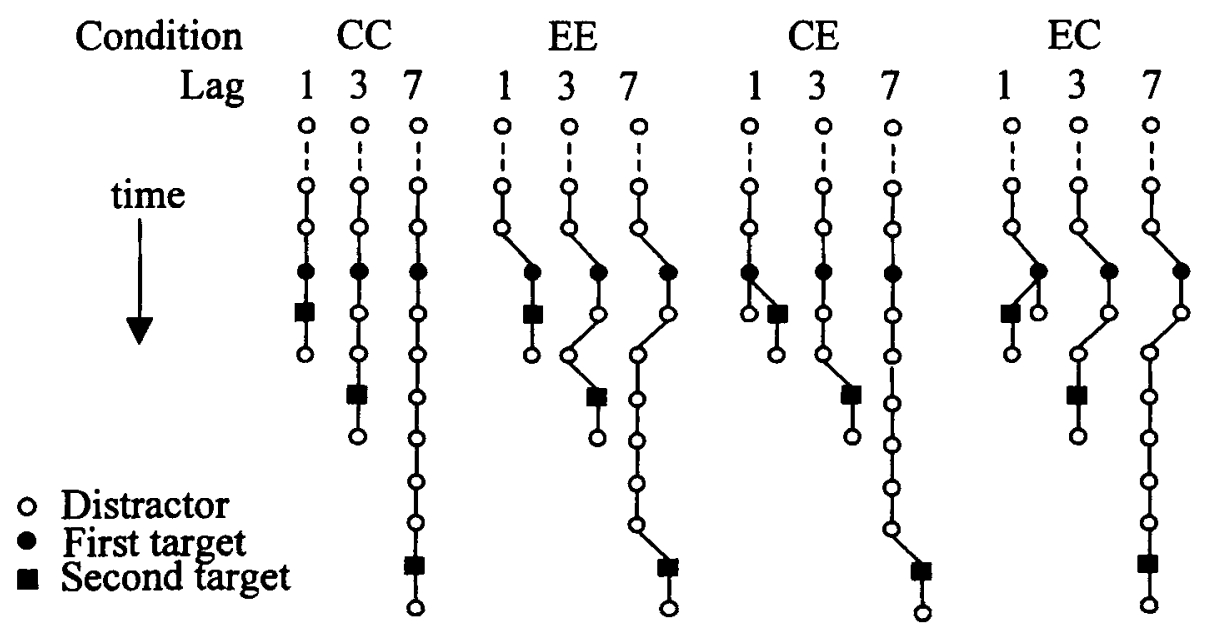

\begin{abstract}
Figure 1. Schematic representation of the stimulus sequence in each condition at Lags 1,3 , and 7. The segmented line at the beginning of each sequence represents the set of 7-10 leading distractors that were displayed at the center of the screen before the first target on each trial. The symbols shown as displaced from the center line represent items that were displayed eccentrically, above, below, to the left of, or to the right of center.
\end{abstract}

\section{RESULTS}

Estimates of T2 identification were based exclusively on the trials in which $\mathrm{T} 1$ had been identified correctly. This procedure is commonly adopted in $\mathrm{AB}$ experiments on the grounds that, on incorrect trials, the source of error is unknown, so the effect of $\mathrm{Tl}$ on the processing of T2 cannot be estimated. Responses were recorded as correct regardless of the order of report. Mean percentages of correct identifications of $T 1$ were 89,90,90, and 90 for Conditions $\mathrm{CC}, \mathrm{CE}, \mathrm{EC}$, and EE, respectively. Mean

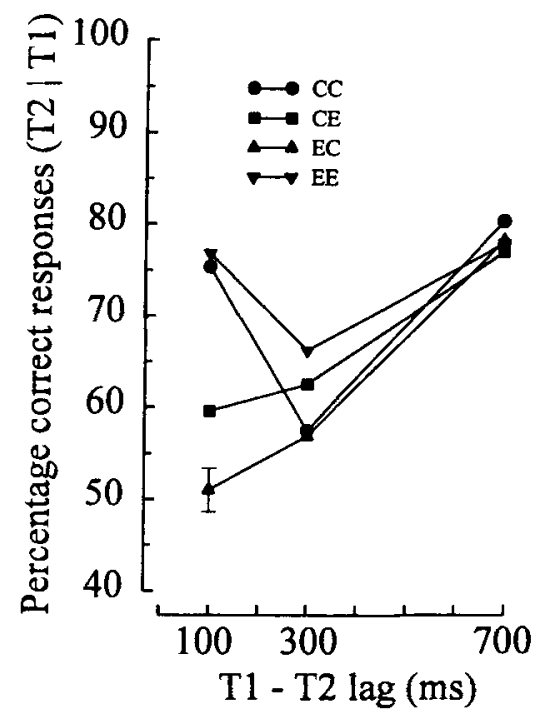

Figure 2. Mean percentage of correct identification of the second target, given that the first target had been identified correctly. The four conditions were central-central $(\mathrm{CC})$, central-eceentric (CE), eccentric-central (EC), and eccentric-eccentric (EE). The error bar represents the average standard error of the mean. percentages of correct identifications of $\mathrm{T} 2$ as a function of lag are presented in Figure 2, separately for the four conditions. The results were analyzed in a 4 (shift: $\mathrm{CC}, \mathrm{CE}$, $\mathrm{EC}$, and $\mathrm{EE}) \times 3$ (T1-T2 lag: 100, 300, and $700 \mathrm{msec})$ repeated measures analysis of variance. The analysis revealed significant effects of shift $[F(3,69)=10.73$, $\left.M S_{\mathrm{e}}=705.94, p<.001\right], \operatorname{lag}\left[F(2,46)=34.01, M S_{\mathrm{e}}=\right.$ $930.39, p<.001]$, and a shift $\times$ lag interaction $[F(6,138)=$ $\left.16.30, M S_{\mathrm{e}}=291.57, p<.001\right]$. Individual $t$ tests performed on the results for Lag 1 revealed that Condition CE differed significantly from Condition EC $[t(190)=$ $3.55, p<.001]$. Individual $t$ tests performed on the results for Lag 3 revealed that Condition CE did not differ significantly from $\mathrm{EE}[t(190)=1.42, p>.05]$, but Condition CE differed from EC $[t(190)=2.18, p<.05]$.

The shift $\times$ lag interaction effect was examined further by means of separate polynomial contrast analyses. Because the lag variable had only 2 degrees of freedom, only the linear and the quadratic components could be estimated. The percentages of the total variance attributable to the linear and quadratic components, respectively, in each of the four conditions were as follows. Condition CC, $4.3 \%$ and $95.7 \%$; Condition EE, $0.8 \%$ and $99.2 \%$; Condition CE, $87.2 \%$ and $12.8 \%$; Condition EC, $90.4 \%$ and $9.6 \%$. This confirms the graphical evidence in Figure 2 that the quadratic component was predominant in Conditions $\mathrm{CC}$ and $\mathrm{EE}$, whereas the linear component was predominant in Conditions $\mathrm{CE}$ and $\mathrm{EC}$.

\section{DISCUSSION}

The results are unambiguous: Lag 1 sparing was very much in evidence in Conditions CC and EE, but not in Conditions CE and EC. Although the results are clearcut, the rules by which spatial switching affects identification of $\mathrm{T} 1$ and $\mathrm{T} 2$ are not immediately obvious. It should 
be kept in mind that a spatial switch was never detrimental to identification of $T 1$. Nor was a spatial switch away from the location of the RSVP stream necessarily detrimental to identification of T2, as indicated by the Lag 1 sparing obtained in Condition EE. Yet spatial switching was clearly detrimental for identification of $\mathrm{T} 2$ in Conditions $\mathrm{CE}$ and $\mathrm{EC}$.

This pattern of results can be explained by an elaborated version of the attentional gating hypothesis outlined in the introduction. We assume with Chun and Potter (1995) that processing occurs in two sequential stages. Initial processing takes place in Stage 1, where potential targets are singled out for further processing in Stage 2. Items to be transferred to Stage 2 are channeled through an attentional gate that is said to open rapidly but to close sluggishly in about 150-200 msec (Chun \& Potter, 1995; Shapiro \& Raymond, 1994). Transfers from Stage 1 can take place as long as Stage 2 is not busy processing earlier items.

To account for the present results, we make explicit two assumptions that were largely implicit in the earlier statements of the attentional gating model. First, we assume that the attentional gate is tied to the spatial location of the relevant stimulus. Second, if a gate is currently open, it must first be closed in order for a new gate to be opened elsewhere. In other words, attention must be disengaged from the current location before it can be engaged at a new location (Posner, 1995; Posner \& Raichle, 1994).

Given these assumptions, the results at Lag 1 can be explained as follows:

Condition CC. Lag 1 sparing occurred in Condition CC because, having been presented in rapid succession at the same location, both targets could gain access to Stage 2 through the attentional gate initially opened for T1. Therefore, the two targets gained concurrent access to Stage 2, thus obviating an $\mathrm{AB}$ deficit.

Condition EE. Much the same events as in Condition CC occurred in Condition EE. An attentional gate could be opened readily at the eccentric T1 location, there being no other gate open at the time of $\mathrm{T} 1$ presentation. Because of its spatiotemporal contiguity with T1, T2 passed through the same gate, and Lag 1 sparing ensued.

Condition $C E$. The spatial switch inserted between the presentation of $\mathrm{T} 1$ and $\mathrm{T} 2$ prevented Lag 1 sparing as follows. A gate was opened on arrival of T1 at the central location. Since T2 was presented outside that gate, it remained in Stage 1 until a gate could be opened at the new location. This could not be done until Stage 2 was free after having processed T1. While delayed in Stage 1, T2 remained vulnerable to masking by the trailing item, and an $A B$ deficit ensued.

Condition EC. Lag 1 sparing did not occur in this condition for much the same reason as in Condition CE. An attentional gate at the eccentric location could be opened readily on presentation of $\mathrm{T} 1$ because no other gate was open at that time. This gave $\mathrm{Tl}$ ready access to Stage 2 and immunity from masking. Not so for $\mathrm{T} 2$, which, having been presented outside the attentional gate, was delayed in Stage 1 and remained vulnerable to masking, with a consequent $\mathrm{AB}$ deficit.
Consistent with the present account of Lag 1 sparing based on attentional gating, Lag 1 sparing was not obtained in other studies in which the targets were displaced from the RSVP stream (Breitmeyer et al., 1999; Joseph et al., 1997). Also consistent with the present account are the results obtained with the two-target paradigm, in which the targets are presented in different locations without a central RSVP stream (Duncan et al., 1997; Moore et al., 1996). In these studies, as in the RSVP studies cited above, Lag 1 sparing was notably absent.

One alternative account needs to be considered. The poor performance at Lag 1 in conditions $C E$ and $E C$ could be explained if the observers had made eye movements from the T1 location to the T2 location. Such eye movements would result in saccadic suppression of T2. Although plausible, this account is questionable if we consider the relationship between saccadic latency and the duration of saccadic suppression. The average latency of a saccade is approximately $250 \mathrm{msec}$ (e.g., Heywood \& Churcher, 1980), saccadic suppression being maximal at the time of onset of the eye movement and extending to \pm 100 msec (Volkmann, Riggs, \& Moore, 1980). With these considerations in mind, it is unlikely that saccadic suppression could have impaired identification of $\mathrm{T} 2$ at Lag 1 in the present work. This is because, at Lag 1, T2 followed the onset of T1 by $100 \mathrm{msec}$. If the observer used the onset of $\mathrm{Tl}$ as a signal to initiate a saccade, the actual eye movement would start about $250 \mathrm{msec}$ later. On this timing, saccadic suppression would not begin until about $150 \mathrm{msec}$ after T1, by which time T2 had already been turned off. Clearly, this would put $\mathrm{T} 2$ outside of the period of suppression. At any rate, the saccadic suppression hypothesis is open to empirical testing in future studies by direct monitoring of eye movements.

Our analysis suggests that an elaborated gating hypothesis can best account for the presence of Lag 1 sparing in the present experiment, as well as in related experiments. Still unexplained, however, is the present finding that the level of performance at Lag 1 was significantly lower in Condition EC than in Condition CE. Bearing in mind that the absence of Lag 1 sparing led to relatively low levels of performance in both conditions, an additional factor was probably at work in Condition $\mathrm{EC}$ to lower the level even further. While no definitive account can be given at this stage, the lower level in Condition EC could be related to the number of spatial switches preceding $\mathrm{T} 2$.

As illustrated in Figure 1 (Lag 1 sequence), T2 was preceded by a single spatial switch in Condition CE but by two consecutive switches in Condition EC. Thus, the focus of attention had to be moved twice in rapid succession in Condition EC but only once in Condition CE. This could have impacted on identification of $\mathrm{T} 2$ as follows. In the $\mathrm{CE}$ sequence, opening an attentional gate for T1 did not require a spatial shift because attention was already focused on the central location. Therefore, a gate could be opened readily, and the processing sequence could begin without delay. In contrast, presentation of $\mathrm{T} 1$ in the $\mathrm{EC}$ sequence introduced a delay equivalent to 
the time required to move the focus of attention to the eccentric location, thus postponing the start of the processing sequence. It is likely that the delay could not be absorbed completely within the T1 interval in the RSVP sequence but that it rippled through the next and later intervals, thus delaying the opening of an attentional gate for $\mathrm{T} 2$. This increased the period for which $\mathrm{T} 2$ remained vulnerable to masking in Stage 1, with consequent decrement in accuracy of identification.

Although plausible, this account is post hoc and, therefore, in need of independent verification. Related studies, such as those of Breitmeyer et al. (1999) and Joseph et al. (1997), do not bear on the comparison between Conditions CE and EC because they did not include an EC condition. It is interesting to note, however, that the reduced - but still significant-difference between Conditions CE and EC at Lag 3 (Figure 2) is consistent with a diminishing effect of the delay in Condition EC rippling through successive intervals in the RSVP sequence. One way in which this account may be verified is by introducing a brief delay before presenting T2 at the central location in Condition CE. To the extent that the delayed presentation compensates for the delay introduced by the attentional switch, Lag 1 performance in Condition CE should rise toward the level of Condition EC.

In the present work, we have elucidated the conditions that lead to the occurrence of Lag 1 sparing. We found that Lag 1 sparing occurs when T1 and T2 are presented in the same spatial location, but not when they are presented in different locations. This pattern of results suggests that the presentation of a target triggers the opening of an attentional gate that is tied to the spatial location of that target. This initial gate must be closed (i.e., attention must be disengaged) before a new gate can be opened to process a subsequent target in a different spatial location.

\section{REFERENCES}

Breitmeyer, B. G., Ehrenstein, A., Pritchard, K. K., Hiscock, M., \& CRISAN, J. (1999). The roles of location specificity and masking mechanisms in the attentional blink. Perception \& Psychophysics, 61, 798-809.
ChUn, M. M., \& PotTer, M. C. (1995). A two-stage model for multiple target detection in rapid serial visual presentation. Journal of Experimental Psychology: Human Perception \& Performance, 21, 109-127.

DunCan, J., MarTens, S., \& WARD, R. (1997). Restricted attentional capacity within but not between sensory modalities. Nature, $\mathbf{3 8 7}$, 808-810

DunCan, J., WARD, R., \& ShapiRo, K. L. (1994). Direct measurement of attentional dwell time in human vision. Nature, 369, 313-315.

Herwood, S., \& Churcher, J. (1980). Structure of the visual array and saccadic latency: Implications for oculomotor control. Quarterly Journal of Experimental Psychology, 32, 335-341.

JoLICOEUR, P. (1998). Modulation of the attentional blink by on-line response selection: Evidence from speeded and unspeeded Task deci- $_{1}$ sions. Memory \& Cognition, 26, 1014-1032.

Joseph, J. S., Chun, M. M., \& Nakayama, K. (1997). Attentional requirements in a preattentive feature search task. Nature, 387, 805-808.

Moore, C. M., Egeth, H., Berglan, L. R., \& Luck, S. J. (1996). Are attentional dwell times inconsistent with serial visual search? Psychonomic Bulletin \& Review, 3, 360-365.

Peterson, M. S., \& Juola, J. F. (1997, November). Spatial and temporal dynamics of attentional gating and the attentional blink. Poster session presented at the annual meeting of the Psychonomic Society, Philadelphia

PoSNER, M. I. (1995). Attention in cognitive neuroscience: An overview. In M. S. Gazzaniga (Ed.), The cognitive neurosciences (pp. 615-624). Cambridge, MA: MIT Press.

PoSNer, M. I., \& RaICHLE, M. E. (1994). Images of mind. New York: Scientific American.

Potter, M. C., Chun, M. M., Banks, B. S., \& Muckenhoupt, M. (1998). Two attentional deficits in serial target search: The visual attentional blink and an amodal task-switch deficit. Journal of Experimental Psychology: Learning, Memory, \& Cognition, 24, 979-992.

Raymond, J. E., Shapiro, K. L., \& ARnell, K. M. (1992). Temporary suppression of visual processing in an RSVP task: An attentional blink? Journal of Experimental Psychology: Human Perception \& Performance, 18, 849-860.

ShAPIRO, K. L., \& RAYmond, J. E. (1994). Temporal allocation of visual attention: Inhibition or interference? In D. Dagenbach \& T. H. Carr (Eds.), Inhibitory processes in attention, memory, and language (pp. 151-188). San Diego: Academic Press.

VISSER, T. A. W., BISCHOF, W. F., \& DI LoLlo, V. (in press). Attentional switching in spatial and non-spatial domains: Evidence from the attentional blink. Psychological Bulletin.

Volkmann, F. C., Riggs, L. A., \& Moore, R. K. (1980). Eye blink and visual suppression. Science, 207, 900-902.

WARD, R., DUNCAN, J., \& SHAPIRO, K. (1997). Effects of similarity, difficulty, and nontarget presentation on the time course of visual attention. Perception \& Psychophysics, 59, 593-600.

(Manuscript received July 29, 1998; accepted for publication November 3, 1998.) 